\title{
Assessing Patients' Needs in Greek Intensive Care Units. Validation of a Respiratory Patient Satisfaction Scale
}

\author{
Dimitrios E. Papageorgiou Evridiki Kaba Martha Kelesi Areti Stavropoulou \\ Penelope E. Tziliali Niki Pavlatou Georgia Fasoi \\ ICU Follow- up Care Lab, Department of Nursing, University of West Attica, Athens, Greece \\ E-mail of the corresponding author: dpapa@uniwa.gr
}

\begin{abstract}
Respiratory patients constitute a specific and large category of patients hospitalized in Greek Intensive Care Units (ICUs). These patients have specific needs which differ significantly from other groups of patients treated in general ICUs. Assessing the needs and satisfaction levels of respiratory patients is a crucial issue, related to the quality of care provided in ICU. Many questionnaires have been developed to measure patient satisfaction or patient needs. However, no previous attempt has been made to develop an instrument focused on respiratory patient needs with the appropriate psychometric properties. The aim of this study was to describe the development and evaluation of the psychometric properties of the Respiratory Patient Satisfaction Scale, and its validity and reliability, by ICU respiratory patients' satisfaction. The questionnaire was pilot tested with 20 ICU respiratory patients to identify problems of wording or length of the instrument. Based on feedback, the wording was adjusted and certain items were combined. A new convenience sample of 164 ICU respiratory patients then performed a test-retest of the questionnaire. Internal consistency was assessed using Cronbach alpha coefficients and stability of items was evaluated through test and retest comparison and expressed through intraclass correlation coefficient (ICC). The internal consistency reliability coefficients exceed the minimum 0.50 for Cronbach's coefficient alpha. An exploratory factor analysis revealed seventeen factors, explaining $74.5 \%$ of the variability. Respiratory Patient Satisfaction Scale found to be a comprehensive instrument with satisfactory psychometric properties. The results from this study would serve as references for continuous improvement in intensive care practice helping all patients hospitalized in Greek ICUs and not only the respiratory patients.
\end{abstract}

Keywords: Respiratory patients, Intensive Care Unit, Patient Satisfaction Scale, Validation, Quality care.

DOI: $10.7176 / \mathrm{JEP} / 12-17-01$

Publication date:June $30^{\text {th }} 2021$

\section{INTRODUCTION}

An increasing interest has been evoked during the last years in evaluating and measuring the quality of the health care provided (Chrusch et al. 2016; Topcu et al. 2017). Although health care professionals use outcome measures for assessing the quality of care (Mpinga \& Chastonay 2011; Mosadeghrad 2012) delivered, they neglect that patient satisfaction is a key healthcare provision indicator (Wagner \& Bear 2009; Findik et al. 2010). Patient satisfaction is an outcome measure of patient's health outcomes and confidence in the health system revealing whether or not the care delivered has met the patient's needs and expectations (Beattie et al. 2015; Needleman etal. 2007; Hill et al. 2020).

Lately, the focus on measuring patient satisfaction has increased. Several dimensions of patient satisfaction have been described in the literature as key characteristics that patients experience during their hospitalization (Satyanarayana et al. 2016). The content of these characteristics consisted of the following items: art of care (positively perceived attributes, like friendliness and concern), technical quality of care (for example accuracy, attention to details), interpersonal quality of care, accessibility, physical environment, availability, continuity, efficacy, security, information giving, noise, cleanliness, and food services (Prakash 2010; Ofili 2014).

Studies concerning Intensive Care Units (ICU) have been undertaken aiming mostly to identify the main problems associated with the kind of care patients receive in these settings rather than to evaluate the level of their needs' satisfaction while being hospitalized (Williams \& Leslie 2007; Davidson et al. 2012). To a further extend, issues regarding the quality of the care provided in the Greek ICUs were discussed in recent literature (Koukouli et al. 2018, Stavropoulou et al. 2020). These studies focus mainly on understanding and assessing the Greek family's experiences and needs during critical illness (Koukouli et al. 2018), and on exploring how nurses' perceive the concepts of empathy and empathetic care in high challenge environments, such as ICUs (Stavropoulou et al. 2020).

The present study is a psychometric study testing the reliability and validity of a questionnaire measuring respiratory patients' satisfaction. Respiratory patients constitute a specific and large category of patients hospitalized in an ICU. Mechanical ventilation -invasive or not- is not simply supportive for application of different therapeutic schedules but the main reason for their intensive care hospitalization that provokes a number of problems. This group of patients have specific needs which are different in some degree from those patients of a general ICU. However, no previous attempt has been made internationally to develop an instrument 
to measure respiratory patients' satisfaction receiving intensive care. A considerable effort was made by previous researchers to analyze and measure patients' satisfaction (Abdellah \& Levine 1957a); Risser 1975; Hinshaw \& Atwood 1982; McCormack \& McCance 2006). The present study demonstrates the development of an instrument for respiratory patients in ICU in parallel with the assessment of patients' needs and their satisfaction with the care provided.

\section{AIM}

The aim of this study was to describe the development and evaluation of the psychometric properties of the Respiratory Patient Satisfaction Scale, and its validity and reliability, by measuring ICU respiratory patients' satisfaction.

\section{MATERIALS AND METHODS}

This investigation was conducted within a time period of ten months (between October 2019 and July 2020), in ten hospitals with twelve ICUs varying in capacity from 4 to 12 beds, with an average bed occupancy being over $90 \%$ and an average nurse to patient ratio ranging from $1: 2$ to $1: 3$, depending on patient condition and dependency. Patients $(\mathrm{N}=164)$ participated in the study only once (Table 1). There were 6 inclusion criteria: 1$)$ the participants should be consenting adults older than 18 years old, 2) they should have been in the ICU for at least 4 days, 3) they should be patients facing respiratory problems, 4) they should not be mechanically ventilated at the time of research in order to be able to communicate verbally, 5) they should be in a good mental condition and oriented to person, place and time to participate as judged by the chief physician and 6) they should not be under sedative or tranquilizing medication in order to avoid mental or emotional confusion.

An original pilot instrument was developed composed of 86 questions related to respiratory patients' needs. After completion of 20 pilot interviews, with a representative sample of respiratory patients drawn from ICUs, a second version of the scale was formulated consisting of 72 items describing the following ten dimensions of needs: basic care, communication with staff, technical care, continuity of care, communication with relatives, interpersonal care, ethics, discomforts' relief, participation in decision-making, and effectiveness of care. This version of 72 items derived by the application of the procedure of internal consistency and Cronbach's alpha was used to items reduction. The RPSS ratings on 72 items were made on a four-point scale, ranging from very satisfied, mostly satisfied, slightly satisfied, and not at all satisfied.

\subsection{Statistical analysis}

Data were analyzed with the statistical software program Statistical Package for the Social Sciences (IBM, Athens, Greece) 25.0. Factor analysis based on 164 interviews with Varimax rotation was applied for data reduction. This technique enabled the researchers to see a reduction of a 72-item scale to a set of seventeen factors. In the Varimax rotation matrix the eigenvalues associated with each factor represent the amount of total variance accounted for, by the factor. Loadings were examined on each principal component considered as a factor. The scale's internal consistency with the correlation of each item with the remainder of the scale and coefficient alpha was computed. Pearson's product correlations were estimated between the 72 items of the RPSS for the total sample (correlation matrix). Test - retest reliability of the RPSS was explored by reinter viewing in $48 \mathrm{~h}$ period a random sample of 66 respiratory patients from the initial sample of 164 subjects, and the Pearson's correlations coefficients (r) of each item and the global scale scores between the first and second interview were estimated.

\section{RESULTS}

Unrotated factor structure of the RPSS scale has been explored in two principal component analyses with eigenvalues $>1$. A series of rotations to the Varimax criterion was carried out. The clearest most relevant form was obtained by rotating seventeen factors with associated "eigenvalues" ranging from 3.51 (factor 4) to 1.95 (factor 17), which accounted for $74.5 \%$ of the total variance in the data (Table 2 ).

All seventeen factor internal consistency reliability coefficients exceed the minimum 0.50 for Cronbach's coefficient alpha, so the RPSS scale items could be considered as reliable instrument (Table 3).

Test - retest reliability results of the RPSS have shown all correlation coefficients ( $r$ ) of each item and the global RPSS score between the two interviews ranging from 0.92 to 0.86 at statistically significant levels.

\section{DISCUSSION}

This is instrument developed to evaluate the respiratory patients' needs and their satisfaction with care provided in Greek Intensive Care Units. The validity of the content of this measuring tool was based on the extensive literature review (Beattie et al. 2015; Needleman etal. 2007; Hill et al. 2020; Vermeir et al. 2015; Uijen et al. 2012) and on concepts such as: patient satisfaction (Wagner \& Bear 2009; Findik et al 2010; Gill \& White 2009; Batbaatar et al. 2016); Berkowitz 2016), patient needs, quality of care in general and of ICU care in particular 
(Mpinga \& Chastonay 2011; Mosadeghrad 2012; Williams \& Leslie 2007; Davidson et al. 2012), the research results, which concern the identification of patients' needs (Abdellah \& Levine 1957a; Risser 1975; Hinshaw \& Atwood 1982; McCormack \& McCance 2006; Lawrence et al. 2015; Ampaw et al. 2020; Cinaroglu \& Baser 2016; Keller et al. 2014; Hunt et al. 2013; Gorari \& Theodosopoulou 2015; Agheorghiesel \& Copoeru 2013),the experts who evaluated the measuring tool, the pilot study (sample of 20 respiratory patients) that evaluated the content and the framework of the questionnaire and the quantitative and qualitative data, which were used to develop this measuring. The validity (based on some criteria), which was measured by Pearson's correlation coefficient, was confirmed by the degree of correlation between the questions (in the first section of the questionnaire) and the problems (in the second section of the questionnaire) and their total sum.

The structural validity of the measuring tool was based on factor analysis. The factor analysis highlighted 17 factors which explained $74.5 \%$ of the variability and the heterogeneity of the questionnaire.

The internal consistency reliability of the components of the used measuring tool was measured by the Cronbach's alpha coefficient. According to the results it was found that: Cronbach's alpha was 0.92 for the total 72 questions from the first section, Cronbach's alpha values ranged from 0.52 to 0.95 for the 10 categories of needs (the 72 questions were grouped into these 10 categories), Cronbach's alpha was 0.89 for the total 46 problems (second part) and finally Cronbach's alpha values ranged from 0.67 to 0.96 for the factors that resulted from the factor analysis.

Considering that a Cronbach's alpha value equals to 0.70 is satisfactory, the measuring tool, which was used in the present study, had reliability that ranges from a satisfactory level to a highest level.

\subsection{Limitations}

There are several limitations in this study which must be considered in interpreting its results. The sample size could be considered small for reliability testing since it was a convenient sample from the population of respiratory patients hospitalized in the ICUs where the study was conducted. Such a sampling technique may suggest selection bias. On the other hand, doing research on intensive care settings where patients are very ill and treatment is intense, constitutes a problem as patients are not always available. In the present study, a number of patients were not approached due to their busy treatment plans while others were not able to communicate verbally. The small sample size in this study is a serious problem but might not have affected results and conclusions to such a great extent. Also, respiratory patients who chose to participate even though facing a number of different problems did not seem to have perceptions of intensive care that differ from respiratory patients being in a better state of health. An additional limitation is the fact that the study findings reflected the views of respiratory patients being from only ten hospitals and twelve ICUs of them and might not necessarily represented the perceptions of respiratory patients hospitalized in ICUs all over Greece. Perhaps, some specific organizational factors of intensive care settings studied may have some influence on the present data. Based on the limitations mentioned above, a similar study with a larger sample of both respiratory patients and ICU staff, drawn from a cross-sectional range of Greek institutions, would have greater generalizability and validity.

\subsection{Recommendations for future research}

Applying the Respiratory Patient Satisfaction Scale in a larger sample of respiratory patients treated in ICUs and in medical and chest clinics is recommended. Furthermore, identifying and assessing the needs of patients suffering from respiratory diseases, including chronic obstructive pulmonary disease, at a primary care level, is recommended, as this might provide valuable information for the quality of care provided in the community. Such research evidence is essential for redesigning community - based care, improving the patients' quality of life and supporting patients' autonomy. Future research in the field involving patients from broad geographical areas may also contribute in formulating specific recommendations for self-management of the disease and reducing mortality rates.

\section{CONCLUSIONS}

This study described the process used to develop and implement a patient satisfaction tool in ICU setting in an attempt to assess respiratory patients' needs (physiological, psychological, and social) in Greece as expressed by ICU patients. The psychometric properties of the instrument were satisfactory as an existence of internal consistency reliability, content validity, criterion - related validity and construct validity was found. The Respiratory Patient Satisfaction Scale questionnaire is a valid quality indicator to measure the delivered care as perceived by patients. The instrument can be used as a useful outcome measure in studies that attempt to improve satisfaction with ICU care.

The results from this study would serve as references to continuous improvement in intensive care practice helping all patients hospitalized in Greek ICUs and not only the respiratory patients. 


\section{REFERENCES}

Abdellah, F.G. \& Levine, E. (1957a), Developing a measure of patient and personnel satisfaction with the nursing care, Nursing Research 5, 100-108.

Ampaw, E.M., Chai, J., Liang, B., Tsai, S.B. \& Frempong J. (2020), Assessment on health care service quality and patients' satisfaction in Ghana, Kybernetes 49(12), 1- 23.

Agheorghiesei, D.T. \& Copoeru, I. (2013), Evaluating Patient Satisfaction- A Matter of Ethics in the Context of the accreditation Process of the Romanian Hospitals, Social and Behavioral Science 82, 404-410.

Batbaatar, E., Dorjdagva, J. \& Luvsannyam, A. (2016), Determinants of patient satisfaction: a systematic review, Perspectives in Public Health 20(11), 1- 13.

Beattie, M., Murphy, D.J., Atherton, I. \& Lauder W. (2015), Instruments to measure patient experience of healthcare quality in hospitals: a systematic review, Syst Rev 23(4), 97.

Berkowitz, B. (2016), The Patient Experience and Patient Satisfaction: Measurement of a Complex Dynamic, Online J Issues Nurs 21(1), 1.

Chrusch, C.A., Martin, C.M. \& The Quality Improvement in Critical Care Project. (2016), Quality Improvement in Critical Care: Selection and Development of Quality Indicators, Canadian Respiratory Journal 2016, 2516765.

Cinaroglu, S. \& Baser, O. (2016), Understanding the relationship between effectiveness and outcome indicators to improve quality in healthcare, Total Quality Management 1294-1311.

Davidson, J., Jones, C. \& Bienvenu O.J. (2012), Family response to critical illness: Post intensive care syndrome- family, Crit Care Med 40(2), 618-624.

Findik, U.Y., Unsar, S. \& Sut N. (2010), Patient satisfaction with nursing care and its relationship with patient characteristics, Nursing and Health Sciences 12, 162- 169.

Gorari, A. \& Theodosopoulou, E. (2015), Satisfaction with nursing care provided to patients who have undergone surgery for neoplastic disease, Prog Health Sci 5(1), 29-41.

Gill, L. \& White, L. (2009), A critical review of patient satisfaction, Leadership in Health Services 22(1), 8-19.

Hill, J.E., Stephani, A,M, Sapple, P. \& Clegg A. (2020), The effectiveness of continuous quality improvement for developing professional practice and improving health care outcomes: a systematic review, Implementation Science 15, 23.

Hinshaw, A.S. \& Atwood, J.R. (1982), A patient satisfaction instrument: precision by replication, Nurs Res 31(3), 170-175.

Hunt, K.J, Shlomo, N. \& Addington-Hall J. (2013), Participant recruitment in sensitive surveys: a comparative trial of 'opt in' versus 'opt out' approaches, BMC Med Res Methodol 11(13), 3.

Keller, A.C., Bergman, M.M., Heinzmann, C., Todorov, A., Weber, H. \& Heberer, M. (2014), The relationship between hospital patients' ratings of quality of care and communication, Int J Qual Health Care 26(1), 26-33.

Koukouli, S., Lambraki, M., Sigala, E., Alevizaki, A., \& Stavropoulou, A. (2018), The experience of Greek families of critically ill patients: Exploring their needs and coping strategies. Intensive \& critical care nursing 45, 44-51.

Lawrence, D., Bryant ,T.K., Nobel, T.B., Dolansky, M.A. \& Singh M.K. (2015), A comparative evaluation of patient satisfaction outcomes in an interprofessional student-run free clinic, J Interprof Care 29(5), 445-50.

McCormack, B. \& McCance, T.V. (2006), Development of a framework for person- centered nursing, JAN Leading Global Nursing Research 56(5), 472-479.

Mosadeghrad, A.M. (2012), A Conceptual Framework for Quality of Care, Mater Sociomed 24(4):251-261.

Mpinga, E.K. \& Chastonay, P. (2011), Satisfaction of patients: a right to health indicator? Health Policy, 100(23), 144-50.

Needleman. J., Kurtzman, E.T. \& Kizer, K.W. (2007), Performance Measurement of Nursing Care, Medical Care Research and Review 64(2):10S-43S.

Ofili, U.O. (2014), Patients' satisfaction in healthcare delivery - a review of current approaches and methods, European Scientific Journal 10(25), 1857-1881.

Prakash, B. (2010), Patient Satisfaction, Journal of Cutaneous and Aesthetic Surgery 3(3), 151-155.

Risser, N.L. (1975), Development of an instrument to measure patient satisfaction with nurses and nursing care in primary care settings, Nurs Res 24(1), 45-52.

Satyanarayana, V., Sagi, Cong Chen, Kyaw, Z. Htun, Kalyani Puvanendrampillai, Medhavi Ratnayake, Joseph Ngwira, Jeyanthy Rajkanna \& Samson O. Oyibo. (2016), Patient Experience during Hospital Stay: A Pilot Survey, Health 8(14) 1518- 1528.

Stavropoulou, A., Rovithis, M., Sigala, E., Pantou, S., \& Koukouli, S. (2020), Greek nurses' perceptions on empathy and empathic care in the Intensive Care Unit. Intensive \& critical care nursing 58, 102814. https://doi.org/10.1016/j.iccn.2020.102814

Topcu, S., Alpar, S.E., Gulseven, B. \& Ayda Kebapçı. (2017), Patients' experiences in intensive care units: a systematic review, Patient Experience Journal 4(3), 115-127. 
Uijen, A.A., Schers, H.J., Schellevis, F.G. \& van den Bosch WJ. (2012), How unique is continuity of care? A review of continuity and related concepts, Fam Pract 29(3), 264-71.

Vermeir, P., Vandijck, D., Degroote, S., Peleman, R., Verhaeghe, R., Mortier, E., Hallaert, G., Van Daele, S., Buylaert, W. \& Vogelaers, D. (2015), Communication in healthcare: a narrative review of the literature and practical recommendations, Int J Clin Pract 69(11), 1257-67.

Wagner, D. \& Bear M. (2009), Patient satisfaction with nursing care: a concept analysis within a nursing framework, Leading Global Nursing Research 65(3):692-701.

Williams, T. \& Leslie G.D. (2007), Beyond the walls: A review of ICU clinics and their impact on patient outcomes after leaving hospital, Australian Critical Care 21(1):6-17.

Table1.Socio - demographic and clinical characteristics of the Study Patients $(n=164)$

\begin{tabular}{|c|c|}
\hline Characteristics & $\%$ \\
\hline \multicolumn{2}{|l|}{ Age $(56.4 \pm 17.26)$} \\
\hline$<30$ & 9.2 \\
\hline 30-39 & 10.0 \\
\hline $40-49$ & 13.1 \\
\hline $50-59$ & 16.1 \\
\hline$>60$ & 51.6 \\
\hline \multicolumn{2}{|l|}{ Educational level } \\
\hline Illiterate & 9.7 \\
\hline Primary school & 48.4 \\
\hline High school & 37.1 \\
\hline Technological Institute & 3.2 \\
\hline University & 1.6 \\
\hline \multicolumn{2}{|l|}{ Gender } \\
\hline Male & 59.7 \\
\hline Female & 40.3 \\
\hline \multicolumn{2}{|l|}{ Years of disease $(7.40 \pm 13.59)$} \\
\hline \multicolumn{2}{|l|}{ Occupational status } \\
\hline Public officer & 1.6 \\
\hline Private officer & 9.7 \\
\hline Pensioner & 32.3 \\
\hline Farmer & 8.1 \\
\hline Housekeeper & 17.7 \\
\hline Student & 3.2 \\
\hline Building worker & 9.7 \\
\hline Merchant & 1.6 \\
\hline Independent & 12.9 \\
\hline Naval officer & 1.6 \\
\hline Church official & 1.6 \\
\hline \multicolumn{2}{|l|}{ Residence } \\
\hline Athens & 71 \\
\hline Provinces & 29 \\
\hline None pre-existing illness & 54.8 \\
\hline Admission in the hospital more than once & 32.3 \\
\hline Hospitalized somewhere else & 30.6 \\
\hline Admission in the hospital by ambulance & 50 \\
\hline Venturi Mask used at home & 9.7 \\
\hline Antibiotic therapy & 12.9 \\
\hline \multicolumn{2}{|l|}{ Admission diagnosis } \\
\hline COPD & 33.9 \\
\hline Multiinjured & 17.7 \\
\hline Infections of Respiratory System & 12.9 \\
\hline Bronchial Asthma & 6.5 \\
\hline Other & 29.0 \\
\hline Duration of hospitalization $(20.06 \pm 23.61)$ & \\
\hline
\end{tabular}


Table 2.The seventeen RPSS dimensions

- $\quad$ Factor 1. Included questions on interpersonal relations and communicational competence of staff (ten items) and accounted for the $11.3 \%$ of the variability.

- $\quad$ Factor 2. Included questions on communication with relatives (seven items) and accounted for the $10 \%$ of the variability

- $\quad$ Factor 3. Included questions on technical competence of staff (eight items) and accounted for the $6.6 \%$ of the variability.

- $\quad$ Factor 4. Included questions on respect of patient's rights (three items) and accounted for the $4.5 \%$ of the variability

- $\quad$ Factor 5. Included questions on satisfaction of patient's psycho-sociological problems (four items) and accounted for the $4.3 \%$ of the variability

- $\quad$ Factor 6. Included questions on frequency of patient's information (five items): and accounted for the $4.2 \%$ of the variability

- $\quad$ Factor 7. Included questions on friendly environment (three items) and accounted for the $4.2 \%$ of the variability

- $\quad$ Factor 8 . Included questions on staff's cooperation (four items) and accounted for the $3.9 \%$ of the variability

- $\quad$ Factor 9. Included questions on continuity of care (two items) and accounted for the 3.7\% of the variability

- Factor 10. Included questions on relief of symptoms (two items) and accounted for the $3.6 \%$ of the variability

- $\quad$ Factor 11. Included questions on protection of anonymity (three items) and accounted for the $3.3 \%$ of the variability

- $\quad$ Factor 12. Included questions on realization of patient's emotional problems (one item) and accounted for the $2.9 \%$ of the variability

- $\quad$ Factor 13. Included questions on service's availability (one item) and accounted for the $2.8 \%$ of the variability

- $\quad$ Factor 14. Included questions on facing pain with drugs (one item) and accounted for the $2.5 \%$ of the variability

- $\quad$ Factor 15. Included questions on staff's genuine interest (two items) and accounted for the $2.4 \%$ of the variability

- $\quad$ Factor 16. Included questions on quality of care (one item) and accounted for the $2.3 \%$ of the variability

- $\quad$ Factor 17. Included questions on possibility of patient's resting (one item) and accounted for the $2.2 \%$ of the variability

Table 3. The Respiratory Patient Satisfaction Scale (a) Mean Value (b) Internal Consistency

\begin{tabular}{lcc}
\multicolumn{1}{c}{ Factors } & Mean Value* & Cronbach's a \\
\hline Interpersonal relations and communicational competence of staff & 3.21 & 0.92 \\
Communication with relatives & 3.11 & 0.69 \\
Technical competence of staff & 3.41 & 0.88 \\
Respect to patient's rights & 3.51 & 0.70 \\
Satisfaction of patient's psycho-sociological & 3.40 & 0.77 \\
problems & & 0.78 \\
Frequency of patient's information & 3.12 & 0.68 \\
Friendly environment & 2.73 & 0.83 \\
Staff's cooperation & 3.25 & 0.80 \\
Continuity of care & 3.31 & 0.71 \\
Relief of symptoms & 3.32 & 0.67 \\
Protection of anonymity & 3.12 & 0.96 \\
Realization of patient's emotional problems & 2.37 & 0.96 \\
Service's availability & 2.49 & 0.96 \\
Pain management medication & 3.71 & 0.96 \\
Staff's genuine interest & 3.20 & 0.96 \\
Quality of care & 2.83 & 0.96 \\
Patient relaxation time & 1.95 & \\
\hline The average value for each factor was based on the average values of the items & \\
\hline which constitute each factor. & & \\
\hline
\end{tabular}

